\title{
Chemical Compositions and Antioxidant Activities of Indonesian Citrus Essential Oils and Their Elucidation Using Principal Component Analysis
}

\author{
Euis Julaeha ${ }^{1}$, Kristin Shinta Dewi ${ }^{1}$, Mohamad Nurzaman ${ }^{2}$, Tatang Wahyudi ${ }^{3}$, Tati Herlina ${ }^{1}$, \\ Ari Hardianto ${ }^{1^{*}}$ \\ 1 Department of Chemistry, Faculty of Mathematics and Natural Sciences, Universitas Padjadjaran, \\ Jatinangor, West Java 45363, Indonesia; euis.julaeha@unpad.ac.id (E.J.); kristinshintadewi@gmail.com \\ (K.S.D.); tati.herlina@unpad.ac.id (T.H.); a.hardianto@unpad.ac.id (A.H.) \\ 2 Department of Biology, Faculty of Mathematics and Natural Sciences, Universitas Padjadjaran, Jatinangor, \\ West Java 45363, Indonesia; m.nurzaman@unpad.ac.id (M.N.) \\ 3 Center for Textile, Jl. Jendral Ahmad Yani No. 390, Bandung, West Java 40272, Indonesia; \\ tatangwahyudi@kemenperin.go.id (T.W.) \\ * Correspondence: a.hardianto@unpad.ac.id
}

\begin{abstract}
Citrus essential oils (EOs) have various bioactivities like antioxidants, with many applications. Antioxidant activities depend on the chemical compositions of the EOs, which are affected by climate, soil, and geographical region. Thus, investigations on chemical compositions and antioxidant activities of Citrus EOs in different countries are valuable. In this study, we distilled EOs from peels of Indonesian-grown Citrus, including C. nobilis, C. limon, C. aurantifolia, C. amblycarpa, and Citrus spp. Chemical compositions of EOs were analyzed using Gas Chromatography-Mass Spectrometer (GC-MS), whereas the antioxidant activities were determined by employing 2,2-diphenyl-2-picrylhydrazyl (DPPH) method. Furthermore, principal component analysis (PCA) was applied to elucidate the main contributing compounds for antioxidant activity. The results show that all EOs possess unique chemical characteristics, with limonene as the majority constituent. For antioxidant activities, C. limon and C. amblycarpa EOs are the two strongest, IC 50 values below $7.00 \mu \mathrm{L} / \mathrm{mL}$. PCA approach suggests that $\gamma$-terpinene mainly contributes to the high antioxidant activities of $C$. limon and C. amblycarpa. Moreover, $o$-cymene, thymol, $p$-cymene, and $\alpha$ pharnesene may also be responsible for the antioxidant activity of $C$. limon EO. These results are valuable information for the applications of Citrus EOs as antioxidant sources.
\end{abstract}

Keywords: chemical composition; antioxidant; Citrus; essential oils; Principal Component Analysis.

\section{Introduction}

Citrus is a prominent genus in the Rutaceae family, which consists of 154 genus and 2100 species. Citrus plants widely spread around the globe dan can grow in tropical and subtropical zones $[1,2]$. Common species from this genus are Citrus aurantifolia, C. nobilis, C. amblycarpa, C. limon (C. limonia, C. limonum), C. aurantium sp. C. aurantium, C. sinensis, C. reticulata, C. paradise, C. bergamia, and C. medica [3]. The consumption of Citrus fruits is extensive due to their delicious tastes and beneficial metabolites such as coumarins, flavonoids, limonoids, pectin carotenoids, as well as vitamins A, C, and E. Those metabolites possess a variety of biological activities, including anti-inflammatory, antioxidant, antimutagen, anticarcinogen, and antiaging [1]. Therefore, Citrus fruits have many applications in the fields of food, health, and cosmetics [3].

The Citrus peels contain glands of essential oils (EOs) in their flavedo and cuticula [4,5]. The EOs have various unique aromas that depend on their chemical compositions [6]. Citrus EOs mainly consist of monoterpenes and sesquiterpenes [5]. One example is Limonene, a monoterpene which constitutes around 65.3 to $95.9 \%$ Citrus EOs [7]. Other compounds are $\beta$-pinene, $\alpha$-terpineol, 
terpinen-4-ol, $\beta$-myrcene, $\gamma$-terpinene, $\beta$-phellandrene, $o$-cymene, and citral. These compounds are variables that contribute to the characteristics and bioactivities of Citrus EOs [8].

Citrus EOs have diverse bioactivities, such as antioxidant [6,7]. Antioxidant protects cellular structure and function by scavenging free radicals, inhibiting lipid peroxidation, and preventing other oxidative degradations [1]. Therefore, compounds with antioxidant bioactivities have been widely used as additives in food products [9] and antiaging in cosmetics [3].

Several research groups have performed studies regarding characterizations and antioxidant activities of different Citrus EOs [10-12]. Moosayv and coworkers [12] analyzed the EO of C. limon grown in Iran and found four main compounds: limonene (46.93\%), terpinene $(16.89 \%)$, tricyclene $(6.67 \%)$, and $\beta$-pinene (4.69\%). Gursoy and colleagues [11] reported that the EO from C. nobilis peels contains limonene $(76.77 \%), \gamma$-terpinene $(8.24 \%)$, linalool $(3.01 \%)$, and myrcene $(2.38 \%)$. They suggested limonene as the compound mainly responsible for the antioxidant activity of $C$. nobilis EO. Meanwhile, Frassinetti and coworkers [10] found that among commercial Citrus EOs in Italy, C. limon EO is the most active antioxidant.

Chemical compositions of Citrus EOs in terms of qualitative and quantitative are affected by climate, soil, and geographical region [13]. Meanwhile, chemical compositions of Citrus EOs determine their antioxidant activities [14]. Thus, investigating chemical compositions and antioxidant activities of Citrus EOs from other countries are important research topics. In this present work, we distilled Citrus EOs from peels of C. nobilis, C. limon, C. aurantifolia, C. amblycarpa, and Citrus spp. grown in Indonesia. Chemical compositions of Citrus EOs were analyzed using Gas Chromatography-Mass Spectrometer (GC-MS). Antioxidant activities of all EOs were also determined using 2,2-diphenyl-2-picrylhydrazyl (DPPH) method. We applied principal component analysis (PCA) to elucidate the responsible secondary metabolites for the antioxidant activity. PCA is a powerful tool for exploratory data analysis and has vital roles in many fields, such as drug design [15], chemotaxonomy [16], and metabolite profiling [17]. This study is essential in providing valuable information about the antioxidant activities of various Citrus EOs and the responsible secondary metabolites. This study will help the application of Citrus EOs in many fields, for example, health, food, functional fabric, and cosmetics.

\section{Results and Discussion}

\subsection{Yields of Citrus Essential Oils}

In this present work, we have distilled EOs from five Citrus species. The obtained Citrus EOs are clear liquids and have pale-yellow colors with fresh aromas. The resulting EO yields vary among species, ranging from $0.34-3.70 \%$. The highest EO yield was acquired from the peel of $C$. nobilis $(3.70 \%)$, followed by C. limon $(0.85 \%)$, C. aurantifolia $(0.78 \%)$, C. amblycarpa $(0.65 \%)$, and Citrus spp. $(0.34 \%)$.

Table 1. Yields of EOs from peels of five different Citrus species.

\begin{tabular}{ccccc}
\hline No. & Citrus Peel & Peel Weight (g) & EO Weight (g) & Yield (\%) \\
\hline 1. & C. nobilis & 500 & 18.5 & 3.70 \\
2. & C. limon & 500 & 4.25 & 0.85 \\
3. & C. aurantifolia & 500 & 3.90 & 0.78 \\
4. & C. amblycarpa & 500 & 3.25 & $=0.65$ \\
5. & Citrus spp. & 500 & 1.70 & $=0.34$ \\
\hline
\end{tabular}

Our results are slightly higher than those reported by others $[5,18]$, where yields of EOs are usually between 0.2 and $2.3 \%$. The EO yields depend on plant genetics, climate, soil, geographical location, and storage period [19]. Therefore, the amount of EO obtained may vary even for the same species. 


\subsection{Chemical Composition}

Table 2 shows that the five Citrus EOs have different chemical compositions in terms of qualitative and quantitative (see Figure S1 for the chemical structures). The Citrus EOs contain monoterpene, monoterpenoid, sesquiterpene, and sesquiterpenoid types of secondary metabolites. These secondary metabolite classes sparsely distribute among the five Citrus EOs. Monoterpenes are the dominating secondary metabolites in Citrus EOs, where limonene is the main component. Limonene constitutes 40 to $67 \%$ of chemical compositions of Citrus EOs. Our result is consistent with the literature that limonene is the most abundant secondary metabolite in Citrus EOs [9]. The highest limonene level presents in Citrus spp. (67.76\%), followed by C. limon (54.62\%), C. nobilis (50.06\%), C. amblycarpa (42.23\%), and C. aurantifolia $(40.84 \%)$.

Table 2. Chemical composition of secondary metabolites in the EOs of five Citrus species.

\begin{tabular}{|c|c|c|c|c|c|}
\hline \multirow{2}{*}{ Secondary Metabolite } & \multicolumn{5}{|c|}{ Percentage in EO $(\%)^{1}$} \\
\hline & C. aurantifolia & C. nobilis & C. limon & C. amblycarpa & Citrus spp. \\
\hline \multicolumn{6}{|l|}{ Monoterpenes } \\
\hline$o$-Cymene & 0.67 & - & 4.66 & - & - \\
\hline$p$-Cymene & - & - & 1.46 & - & - \\
\hline$\alpha$-Pinene & 1.60 & - & - & 0.52 & - \\
\hline Camphene & 0.33 & - & - & 0.57 & - \\
\hline$\beta$-Pinene & 0.81 & 3.69 & 0.29 & 5.30 & - \\
\hline Limonene & 40.84 & 50.06 & 54.62 & 42.23 & 67.76 \\
\hline$\beta$-Phellandrene & 0.36 & 0.70 & - & 1.10 & - \\
\hline$\gamma$-Terpinene & 0.80 & - & 4.30 & 2.50 & - \\
\hline (+)-4-Carene & 1.21 & - & - & - & - \\
\hline 2-Carene & 0.20 & - & 0.42 & 1.21 & - \\
\hline$\gamma$-Elemene & 0.46 & - & - & - & - \\
\hline$\beta$-Myrcene & 17.75 & 4.57 & 0.59 & - & 0.72 \\
\hline$\beta$-Ocimene & 0.63 & 1.37 & - & - & 0.70 \\
\hline \multicolumn{6}{|l|}{ Monoterpenoids } \\
\hline Thymol & - & - & 8.76 & - & - \\
\hline Terpinen-4-ol & 1.34 & 1.51 & 1.14 & 11.63 & - \\
\hline$\alpha$-Terpineol & 3.54 & 4.05 & 3.65 & 4.83 & - \\
\hline trans-Carveol & - & 0.75 & - & - & - \\
\hline Limonene oxide & - & - & - & - & 1.53 \\
\hline Citral & 1.00 & - & - & - & 6.15 \\
\hline Citronellal & - & 2.18 & - & - & - \\
\hline Citronellol & - & 0.70 & - & 4.25 & - \\
\hline Geraniol & - & 0.82 & - & - & - \\
\hline \multicolumn{6}{|l|}{ Sesquiterpenes } \\
\hline$\alpha$-Pharnesene & - & - & 0.34 & - & - \\
\hline$\beta$-Bisabolene & 1.47 & - & - & 2.86 & - \\
\hline \multicolumn{6}{|l|}{ Sesquiterpenoid } \\
\hline Caryophyllene & 1.44 & - & - & - & - \\
\hline
\end{tabular}

1 The levels of secondary metabolites are originated from the corresponding peak area percentages in GC chromatograms, which also consist of primary metabolite peaks (data not shown). 
Every $\mathrm{EO}$ has its chemical composition characteristics. $C$. aurantifolia $\mathrm{EO}$ contains the most diverse secondary metabolites (Table 2). Based on our results, the EO of C. aurantifolia is characterized by seventeen secondary metabolites, which are dominated by monoterpenes as many as twelve compounds. Additionally, the EO has secondary metabolites exclusive to $C$. aurantifolia. These consist of $\gamma$-elemene and (+)-4-carene monoterpenes, as well as a caryophyllene sesquiterpenoid. The EO of $C$. nobilis contains monoterpene and monoterpenoid secondary metabolites, which spread evenly (Table 2). The EO has unique compounds: trans-carveol, citronellal, and geraniol. Like $C$. aurantifolia, C. limon produced EO with chemical composition dominated by monoterpenes. The EO contains three aromatic compounds, including o-cymene, $p$-cymene, and thymol. The two last compounds and $\alpha$-pharnesene are exclusive to C. limon EO. Likewise, $C$. amblycarpa EO contains monoterpenes as the main types of secondary metabolites. The EO has a relatively high content of terpinen-4-ol and citronellol compared to the other Citrus EOs (Table 2). Meanwhile, Citrus spp. EO has the lowest diversity of secondary metabolites. Only four secondary metabolites constitute Citrus spp. EO. They are limonene, $\beta$-myrcene, $\beta$-ocimene, limonene oxide, and citral. The levels of limonene and citral are the highest in Citrus spp. EO. Furthermore, limonene oxide is limited to this EO.

\subsection{Antioxidant Activity}

Antioxidant activities of Citrus EOs are represented by the percentage of free radical scavenging (\%) (Figure S2) and the concentration of 50\% scavenging (Table 2). Each EO exhibits a different inhibition profile in scavenging DPPH free radicals (Figure S2). The EO of C. limon shows the highest antioxidant activity with $\mathrm{IC}_{50}$ of $4.25 \pm 0.08 \mu \mathrm{L} / \mathrm{mL}$ (Table 2), consistent with the work published by Frassinetti and colleagues [10]. The antioxidant activity of $C$. limon EO even higher than that of ascorbic acid. In the second place, $C$. amblycarpa shows an IC $\mathrm{C}_{50}$ value of $6.30 \pm 0.04 \mu \mathrm{L} / \mathrm{mL}$. The EOs of C. aurantifolia and Citrus spp. display similar calculated IC50 values, $12.85 \pm 0.20$ and $13.29 \pm 0.04$ $\mu \mathrm{L} / \mathrm{mL}$, respectively. Meanwhile, the EO of $C$. nobilis possesses the weakest antioxidant activity with an $\mathrm{IC}_{50}$ value of $15.83 \pm 0.04 \mu \mathrm{L} / \mathrm{mL}$. These different antioxidant activities of EOs may be due to their chemical composition characteristics.

Table 2. Antioxidant activities of EOs from five Citrus species.

\begin{tabular}{cc}
\hline Citrus & $\mathrm{IC}_{50}(\boldsymbol{\mu L} / \mathrm{mL})$ \\
\hline Ascorbic Acid & $5.28 \pm 0.02$ \\
C. limon & $4.25 \pm 0.08$ \\
C. amblycarpa & $6.30 \pm 0.04$ \\
C. aurantifolia & $12.85 \pm 0.20$ \\
Citrus spp. & $13.29 \pm 0.04$ \\
C. nobilis & $15.83 \pm 0.04$ \\
\hline
\end{tabular}

\subsection{Principal Component Analysis}

As discussed above, we have identified twenty-five secondary metabolites, which are sparsely distributed among the EOs from five different Citrus species. Those secondary metabolite profiles may contain redundant information, which raises an issue in elucidating antioxidant activities of Citrus EOs. Therefore, we transformed Table 1 into a matrix (Table S1) and performed a PCA approach. Through linear combinations, PCA transformed correlated/uncorrelated descriptors, including twenty-five secondary metabolites. Subsequently, the essential information was extracted into only four uncorrelated variables or principal components (PCs) (Figures 1 and 2).

As portrayed in Figure 1, PC1 is important for camphene, limonene, $\beta$-phellandrene, terpinen4-ol, and $\beta$-bisabolene. PC1 has representation qualities above $70 \%$ for these secondary metabolites (Table S2). Additionally, $\beta$-pinene, $\alpha$-terpineol, 2-carene, and citronellol are represented around $60 \%$ by PC1. The importance of PC2 is very high for $\gamma$-terpinene, above $94 \%$, while for $o$-cymene $63 \%$. For $\beta$-ocimene, thymol, $p$-cymene, and $\alpha$-pharnesene, the representation qualities of PC2 are around $70 \%$. 
Thus, the fifteen secondary metabolites, which are shaded by gradient colors of dark blue in Figure 1, have good representations on PC1 and 2. Meanwhile, PC3 is important for (+)-4-carene, caryophyllene, and $\gamma$-elemene, with representation qualities of $71 \%$. For $\alpha$-pinene and $\beta$-myrcene, the representation qualities of $\mathrm{PC} 3$ are 58 and $51 \%$, respectively.

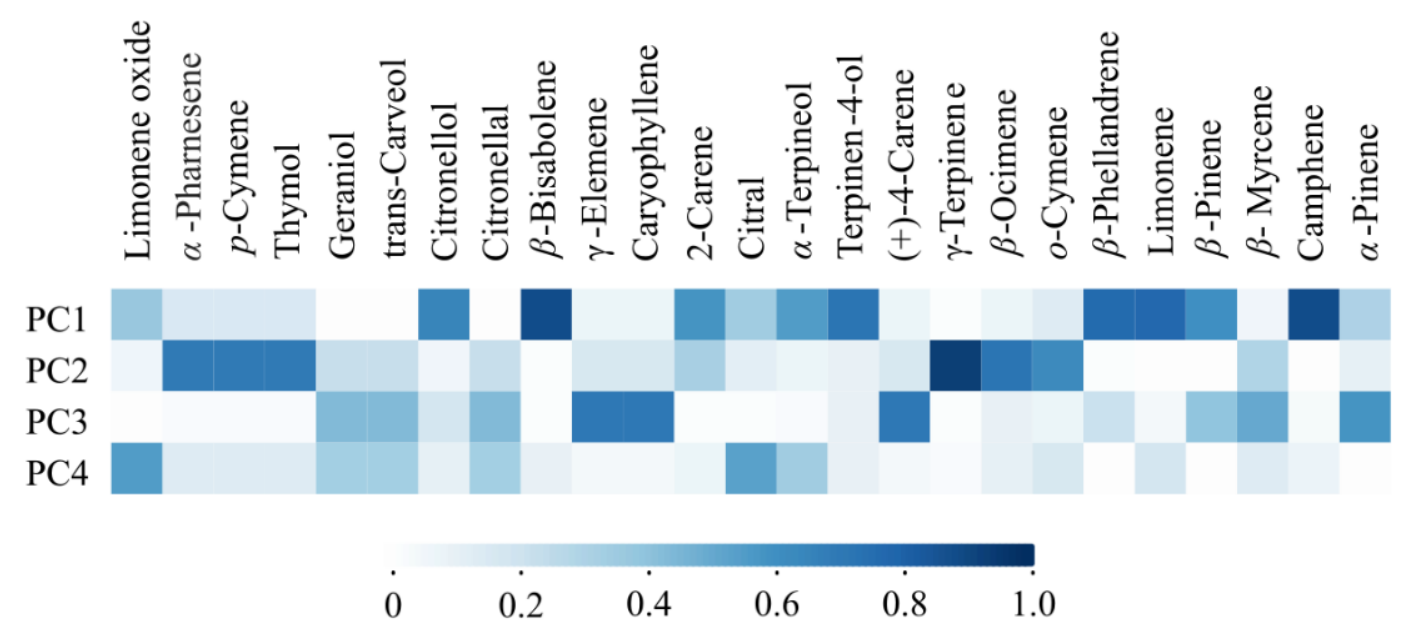

Figure 1. Representation quality heatmap of the descriptors on the PCs. The gradient colors of blue denote scales of representation quality. Representation quality refers to the importance of a principal component for a given variable.

According to Figure 2.a., the proportion of variance explained by each PC is not highly different. PC1 captured $33.8 \%$ of the total variance, while PC2 and 3 explain 26.9 and $23.1 \%$, respectively. The rest variance is described as much as $16.3 \%$ by PC4. Combination of PC1 and 2 only explain $60.7 \%$ of the total variance (Figure 2.b). Thus, we may need at least three PCs to explain more than $80 \%$ of the total variance contributed by secondary metabolite descriptors.

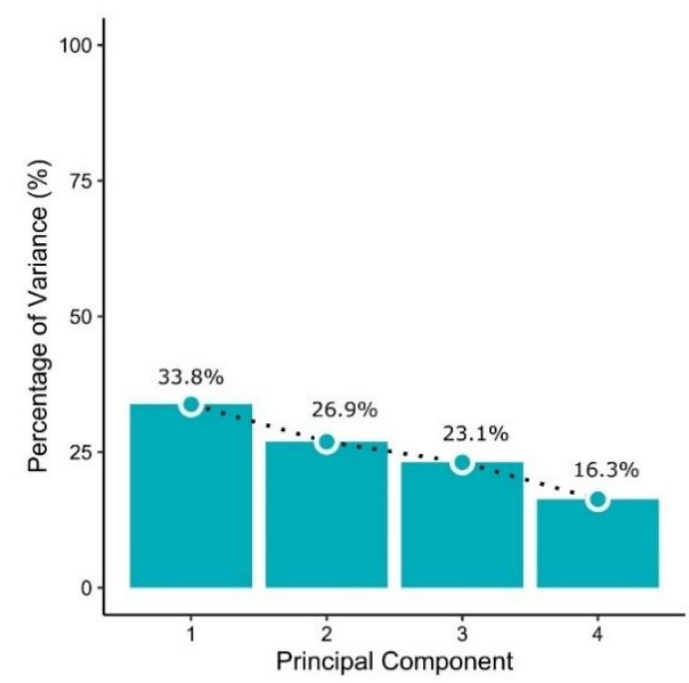

(a)

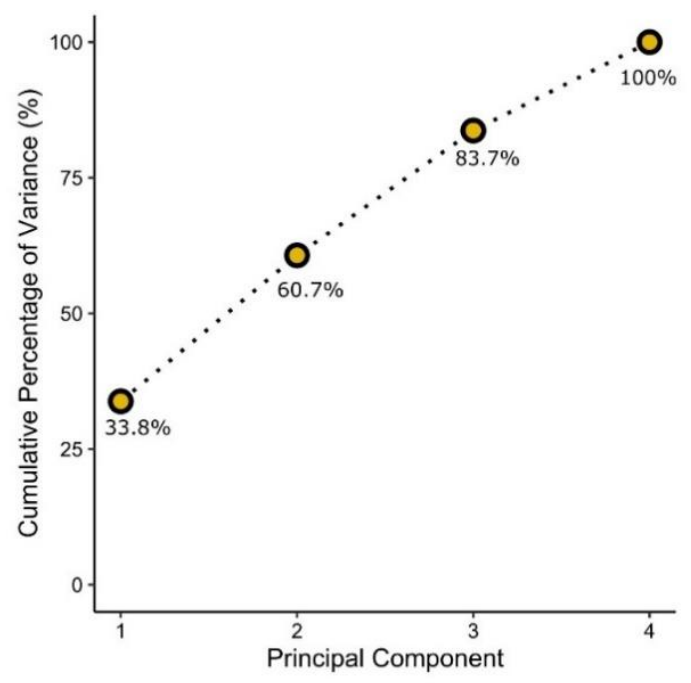

(b)

Figure 2. (a) Scree and (b) cumulative plots of principal component analysis of five Citrus essential oils.

Intriguingly, the score plot on the coordinate system of $\mathrm{PC} 1$ and 2 revealed an informative result (Figure 3.a). In such a coordinate system, Citrus EOs spread according to their antioxidant activities. The EOs with $\mathrm{IC}_{50}$ above $12 \mathrm{ppm}$ (Table S3) cluster in the third and fourth quadrants. On the other hand, the EOs with $\mathrm{IC}_{50}$ around $5 \mathrm{ppm}$ are on the opposing side. The score plot (Figure 3.a) displays 
that the PC2 alone, which explains $26.9 \%$ of the total variance, can separate the essential oils based on their antioxidant activities. Nevertheless, the presence of PC1 assists in further separation of Citrus spp., C. nobilis, and C. aurantifolia. Furthermore, PC1 also helps the analysis through a biplot (Figure 3.b).

The biplot (Figure 3.b) explains the difference between Citrus EOs based on their secondary metabolites. As an example, $C$. limon EO is characterized by thymol, $o$-cymene, $p$-cymene, $\alpha$ pharnesene, and $\gamma$-terpinene. Thymol, $p$-cymene, and $\alpha$-pharnesene present only in $C$. limon EO, whereas the levels of $o$-cymene and $\gamma$-terpinene in the EO are the highest among other Citrus EO. For C. amblycarpa EO, camphene, terpinen-4-ol, $\alpha$-terpineol, 2 -carene, $\beta$-bisabolene, and citronellol describe the oil characteristic. In C. amblycarpa EO, the levels of those secondary metabolites are relatively higher than in other Citrus EOs. Interestingly, the biplot result supports the chemical composition characteristics of EOs in the sub-section on Chemical Composition.

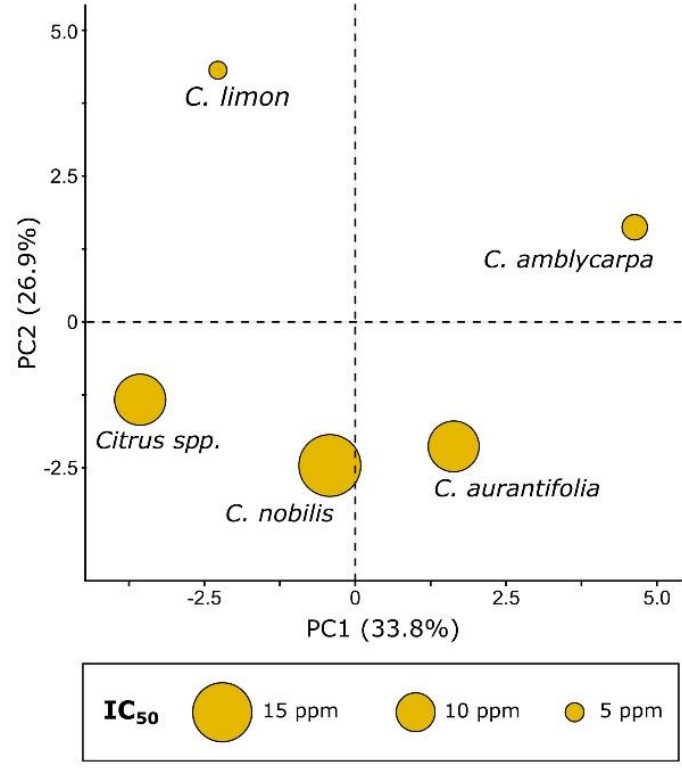

(a)

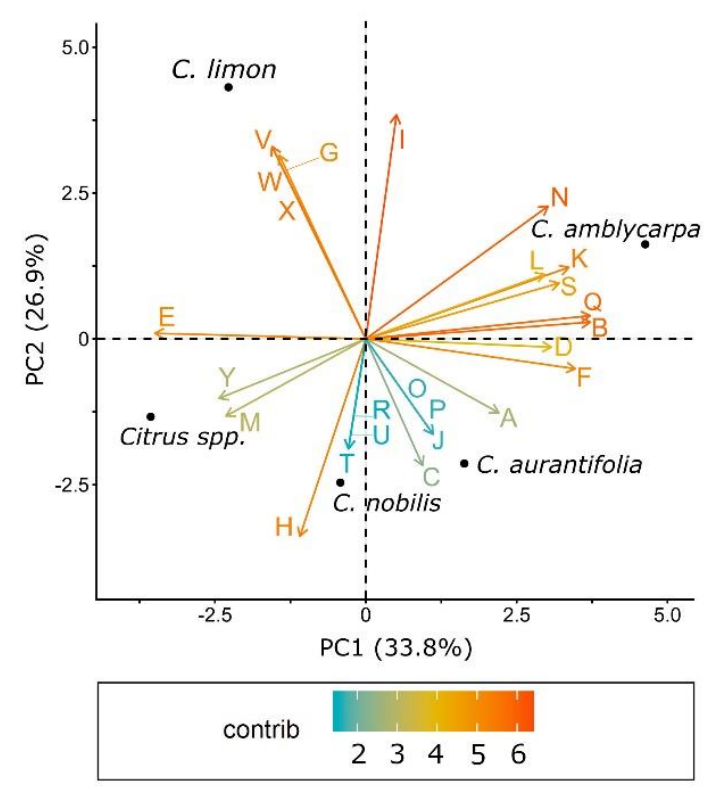

(b)

Figure 3. (a) Individual plot and (b) biplot of EOs from five citrus species in the coordinate system of PC1 and 2. The size of circle in the individual plot represents the value of IC 50. The color gradient and arrow length of the secondary metabolite descriptors in the biplot indicates their importance in the coordinate system of PC1 and PC2. In the biplot, secondary metabolites are coded by alphabetic letters; A: $\alpha$-Pinene; B: Camphene; C: $\beta$-Myrcene; D: $\beta$ Pinene; E: Limonene; F: $\beta$-Phellandrene; G: $o$-Cymene; H: $\beta$-Ocimene; I: $\gamma$-Terpinene; J: $(+)-$ 4-Carene; K: Terpinen-4-ol; L: $\alpha$-Terpineol; M: Citral; N: 2-Carene; O: Caryophyllene; P: $\gamma$ Elemene; Q: $\beta$-Bisabolene; R: Citronellal; S: Citronellol; T: trans-Carveol; U: Geraniol; V: Thymol; W: $p$-Cymene; X: $\alpha$-Pharnesene; Y: Limonene oxide.

As discussed above regarding the score plot (Figure 3.a.), only PC2 can separate Citrus EOs on the factor map based on antioxidant activity (Figure S1). Therefore, in the context of antioxidant activity, we only consider secondary metabolites that are highly represented by PC2 (Figure 1). These secondary metabolites are $\gamma$-terpinene, $\beta$-ocimene, thymol, $o$-cymene, $p$-cymene, and $\alpha$-pharnesene. Not surprisingly, C. limon EO possesses the highest antioxidant activity among others since its character is determined by $\gamma$-terpinene, $o$-cymene, thymol, $p$-cymene, and $\alpha$-pharnesene. The last three secondary metabolites present only in C. limon EO. Additionally, the levels of $\gamma$-terpinene $(4.30 \%)$ and $o$-cymene $(4.66 \%)$ in C. limon EO are the highest, among others. In the EOs with low antioxidant activities, $\gamma$-terpinene and $o$-cymene are absent, or the levels are low (Table S1 and S3). Interestingly, the EO of C. amblycarpa with the second-highest antioxidant activity does not have $o$ cymene, thymol, $p$-cymene, and $\alpha$-pharnesene but contains a considerable amount of $\gamma$-terpinene 
$(2.50 \%)$. Hence, $\gamma$-terpinene may mainly contribute to the high antioxidant activities of $C$. limon and C. amblycarpa. For C. limon EO, its high antioxidant activity may also be supported by o-cymene, thymol, $p$-cymene, and $\alpha$-pharnesene.

\subsection{Chemical Structures of the Antioxidants}

Our study using PCA suggests that thymol, $o$-cymene, $p$-cymene, $\beta$-ocimene, $\alpha$-pharnesene, $\gamma$ terpinene (Figure 4 ) are the metabolites contributing to the antioxidant activities, particularly for $C$. limon EO. Thymol has antioxidant activity because it is a phenolic compound. Its hydroxyl group inhibits DPPH • radicals through hydrogen atom transfer (HAT). After transferring its hydrogen atom, thymol becomes a stable radical (thymol $\bullet$ ) and reacts with other radicals to form DPPH-thymol or thymol-thymol [20].<smiles>Cc1ccc(C(C)C)c(O)c1</smiles>

Thymol<smiles>CC(C)c1ccc(I)cc1</smiles>

p-Cymene

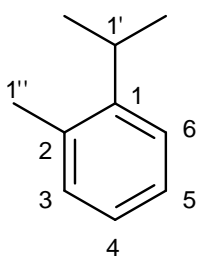

$o$-Cymene

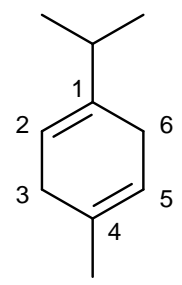

$\gamma$-Terpinene

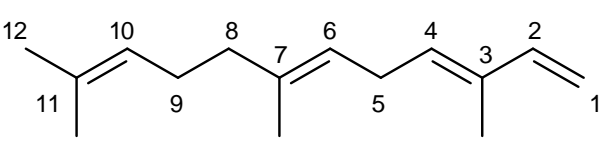

$\alpha$-Pharnesene

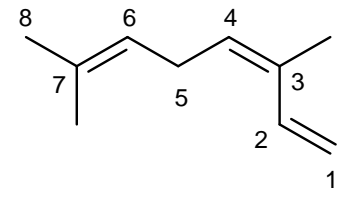

$\beta$-Ocimene

Figure 4. Two-dimensional structures of secondary metabolites responsible to antioxidant activities of EOs according to PCA.

Antioxidant activities of $p$-cymene and $o$-cymene come from their benzylic hydrogen atoms at two different positions, carbon 1' and 1" (Figure 4) [21]. The abstraction of the benzylic hydrogen atoms quenches free radicals, while the secondary metabolites turn into stable radical molecules. In both $\alpha$-pharnesene and $\beta$-ocimene, hydrogen atom transfer from carbon 5 to DPPH $\bullet$ molecules yield stable radicals. The stabilization comes from conjugated double bonds in $\alpha$-pharnesene and $\beta$ ocimene structures, at carbon 1 and 3.

$\gamma$-Terpinene has antioxidant activity even it does not have conjugated double bonds [22]. It is a strong antioxidant similar to $\alpha$-tocopherol, a well-known antioxidant [23]. Liu and Li [24] reported that $\gamma$-terpinene can directly scavenge free radical by donating its hydrogen atom at the allylic position, particularly carbon 3 and 6 (Figure 6). Additionally, $\gamma$-terpinene has been reported to retard linoleic acid peroxidation [22]. Such activity undergoes through a rapid cross-reaction between hydroperoxyl $(\mathrm{HOO} \bullet)$ and linoleylperoxyl $(\mathrm{LOO} \bullet)$ which terminates free radical chain reaction. Moreover, the retardation of linoleic acid peroxidation converts $\gamma$-terpinene to $p$-cymene, an antioxidant.

\section{Materials and Methods}

\subsection{Citrus Essential Oil Preparation and Gas Chromatography-Mass Spectrophotometry}

Fruits of $C$. aurantifolia, C. nobilis, C. limon, C. amblycarpa, and Citrus spp. were collected from Caringin Central Market, Bandung, West Java, Indonesia during July to August 2018. The peels of $C$. aurantifolia, C. nobilis, C. limon, C. amblycarpa, and Citrus spp. were separately cleaned and cut into \pm 2 
$\mathrm{cm}$. Each sample of Citrus peel $(500 \mathrm{~g})$ was subjected into a Clevenger-type hydrodistillation for 8 hours. The obtained EOs were dried using anhydrous sodium sulfate and stored in airtight containers at $4{ }^{\circ} \mathrm{C}$ before gas chromatography-mass spectrometry (GC-MS) analysis.

\subsection{Gas Chromatography-Mass Spectrophotometry (GC-MS)}

Chemical compositions of EOs were analyzed using GC-MS (Agilent GC Type 7890A amd MS Type 5975C). The analysis utilized DB 35MS column with length of $35 \mathrm{~m}$, internal diameter of 0.25 $\mathrm{mm}$, and film thickness of $0.25 \mu \mathrm{m}$. Nitrogen was used as the carrier gas at a flow rate of $1 \mathrm{~mL} / \mathrm{min}$. Every citrus EO was diluted in n-hexane to achieve concentration of $0.5 \%(\mathrm{v} / \mathrm{v})$. Each one microliter EO solution was loaded to an injector with a temperature of $250{ }^{\circ} \mathrm{C}$. The column was initially held as $50{ }^{\circ} \mathrm{C}$ for 1 minute and increased to $250{ }^{\circ} \mathrm{C}$ with a heating ramp of $3.5^{\circ} \mathrm{C} / \mathrm{min}$.

\subsection{Antioxidant Activity Assay}

Antioxidant activities of Citrus EOs were determined using 2,2-diphenyl-2-picrylhydrazyl (DPPH) assay with a modification [25]. Briefly, $0.1 \mathrm{~mL}$ EOs at different concentrations (Table S3) were mixed with $2 \mathrm{~mL} \mathrm{DPPH} \mathrm{(0.21} \mathrm{mM} \mathrm{in} \mathrm{ethanol} \mathrm{95 \% ),} \mathrm{in} \mathrm{duplicate,} \mathrm{and} \mathrm{incubated} \mathrm{in} \mathrm{the} \mathrm{dark} \mathrm{for} 60 \mathrm{~min}$. After incubation, the absorbance was measured at $517 \mathrm{~nm}$. As the control, ethanol was used instead of EO. Ascorbic acid was employed as the standard antioxidant compound. DPPH free radical scavenging activity was computed by utilizing the equation below:

$$
\% D P P H-\text { scavenging activity }=\frac{A_{\text {control }}-A_{\text {sample }}}{A_{\text {control }}} \times 100 \%
$$

$A_{\text {control }}$ denotes control absorbance, whereas $A_{\text {sample }}$ is sample absorbance. Antioxidant activity was also expressed as $\mathrm{IC}_{50}$ or concentration of $\mathrm{EO}(\mathrm{mg} / \mathrm{mL})$ required to inhibit $50 \%$ DPPH. IC 50 was determined from the plot \% DPPH scavenging activity $v$ s. EO concentration.

\subsection{Principal Component Analysis}

Peaks of secondary metabolites, from the GC-MS chromatogram of every citrus essential oil, were manually curated and assigned to a matrix (row $i$, column $k$ ) as listed in Table S1. The citrus species were treated as observation $(i)$, whereas the secondary metabolites such as $\alpha$-pinene and camphene were descriptors $(k)$. Two types of preprocessing were performed to the matrix. The first one was mean centering to retain only the informative variation. The second one was scaling since levels of secondary metabolites in five citrus essential oils displaying different scales.

The matrix was subjected to an orthogonal linear transformation to generate a new coordinate system of principal components [26]. On the axis of rank $s, F_{s}$ (resp. $G_{s}$ ) indicates the coordinate vectors of the citrus species (resp. descriptors). They can be expressed as:

$$
\begin{gathered}
F_{S}(i)=\frac{1}{\sqrt{\lambda_{s}}} \sum_{k} x_{i k} m_{k} G_{S}(k) \\
G_{S}(k)=\frac{1}{\sqrt{\lambda_{s}}} \sum_{k} x_{i k} p_{i} F_{S}(i)
\end{gathered}
$$

$F_{s}(i)$ represents the coordinate of each $i$ on the axis $s$, whereas $G_{s}(k)$ is the coordinate of descriptor $k$ on the axis $s$. Notation $s$ the eigenvalue corresponds to the axis $s, m_{k}$ the weight corresponds to descriptor $k, p_{i}$ is the weight corresponding to each i, $x_{i k}$ is the general term of the matrix (row $i$, column $k$ ). 
Computations and data visualizations were performed in the $\mathrm{R}$ programming language environment [27]. PCA was computed using FactorMineR [26]. Subsequently, the results were visualized using factoextra [28] or ggplot2 [29].

\section{Conclusions}

In this study, we distilled EOs from the peels of C. nobilis, C. limon, C. aurantifolia, C. amblycarpa, and Citrus spp. grown in Indonesia. All EOs contain limonene as the main constituent, ranging from 40 to $67 \%$. Additionally, each EO exhibits individual chemical characteristics. Caryophyllene, (+)-4carene, and c-elemene only present in C. aurantifolia EO, whereas citronellal, trans-carveol, and geraniol are unique metabolites in C. nobilis EO. Thymol, $p$-cymene, and $\alpha$-pharnesene are exclusive in the $\mathrm{EO}$ of $C$. limon. Meanwhile, limonene oxide only presents in the EO of Citrus spp.

The antioxidant assay using DPPH method showed C. limon EO possessing the strongest freeradical-scavenging activity ( $\mathrm{IC}_{50}$ of $4.25 \pm 0.08 \mu \mathrm{L} / \mathrm{mL}$ ), followed by C. amblycarpa $(6.30 \pm 0.04 \mu \mathrm{L} / \mathrm{mL}$ ). Meanwhile, EOs of C. aurantifolia, Citrus spp., and C. nobilis exhibit IC50 values above $12.85 \mu \mathrm{L} / \mathrm{mL}$. Exploratory data analysis using PCA lead to secondary metabolites that may mainly contribute to antioxidant activities of Citrus EOs. These metabolites are thymol, $o$-cymene, $p$-cymene, $\beta$-ocimene, $\alpha$-pharnesene, $\gamma$-terpinene. Monoterpene of $\gamma$-terpinene may play a key role to the high antioxidant activities of $C$. limon and C. amblycarpa EOs. For C. limon EO, its high antioxidant activity may also be contributed by $o$-cymene, thymol, $p$-cymene, and $\alpha$-pharnesene.

In summary, this study provides valuable data regarding antioxidant activities of five Citrus EOs grown in Indonesia and the responsible secondary metabolites. The information will guide the implementations of Citrus EOs as antioxidant sources in many fields, ranging from food, cosmetics, health to functional fabric.

Supplementary Materials: The following are available online at www.mdpi.com/xxx/s1, Figure S1: Chemical structures of secondary metabolites in essential oils (EOs) of five Indonesian-grown Citrus, Figure S2: Percentage of free-radical scavenging activity of five Indonesian-grown Citrus, Figure S3: Score plots on the coordinate systems of all PC combinations, Table S1: A matrix containing five rows of Citrus species and twenty-five columns of secondary metabolites, Table S2: Representation quality of every descriptor on each PC.

Author Contributions: Conceptualization, Euis Julaeha, Mohamad Nurzaman, Tatang Wahyudi, Tati Herlina and Ari Hardianto; Data curation, Euis Julaeha, Kristin Shinta Dewi and Ari Hardianto; Formal analysis, Euis Julaeha and Ari Hardianto; Funding acquisition, Tati Herlina; Investigation, Euis Julaeha, Kristin Shinta Dewi and Mohamad Nurzaman; Methodology, Euis Julaeha, Tatang Wahyudi, Tati Herlina and Ari Hardianto; Software, Ari Hardianto; Validation, Euis Julaeha, Tatang Wahyudi and Tati Herlina; Visualization, Ari Hardianto; Writing - original draft, Euis Julaeha, Kristin Shinta Dewi and Ari Hardianto; Writing - review \& editing, Tati Herlina and Ari Hardianto.

Funding: This research was funded by Kemenristekdikti Penelitian Tesis Magister, grant number 1827/UN6.3.1/LT/2020. The article processing charge (APC) was funded by Universitas Padjadjaran Academic Leadership Grant (ALG), grant number 1397/UN6.3.1/PM/2020.

Acknowledgments: We would like to thank to Kemenristekdikti Penelitian Tesis Magister and Universitas Padjadjaran for financial support.

Conflicts of Interest: "The authors declare no conflict of interest."

\section{Abbreviations}

EO Essential Oil

DPPH diphenyl-2-picrylhydrazyl

GC-MS Gas Chromatography-Mass Spectrometer

IC50 Inhibition Concentration 50; concentration of $\mathrm{EO}(\mathrm{mg} / \mathrm{mL})$ required to inhibit 50\% DPPH.

PCA Principal Component Analysis

PC Principal Component

\section{References}

1. Zou, Z.; Xi, W.; Hu, Y.; Nie, C.; Zhou, Z. Antioxidant activity of Citrus fruits. Food Chemistry 2016, 196, 885-896, doi:https://doi.org/10.1016/j.foodchem.2015.09.072. 
2. Wei, L.; Xiang, X.; Wang, Y.; Li, Z. Phylogenetic relationships and evolution of the Androecia in Ruteae (Rutaceae). PLOS ONE 2015, 10, e0137190.

3. Klimek-Szczykutowicz; Szopa; Ekiert Citrus limon (lemon) phenomenon-A Review of the chemistry, pharmacological properties, applications in the modern pharmaceutical, food, and cosmetics industries, and biotechnological studies. Plants 2020, 9, 119, doi:10.3390/plants9010119.

4. Enejoh, O.S.; Ogunyemi, I.O.; Bala, M.S.; Oruene, I.S.; Suleiman, M.M.; Ambali, S.F. Ethnomedical importance of Citrus aurantifolia (Christm) swingle. The Pharma Innovation Journal 2015, 4, 1-6.

5. Mahato, N.; Sharma, K.; Koteswararao, R.; Sinha, M.; Baral, E.R.; Cho, M.H. Citrus essential oils: Extraction, authentication and application in food preservation. Critical Reviews in Food Science and Nutrition 2019, 59, 611-625, doi:10.1080/10408398.2017.1384716.

6. Teixeira, B.; Marques, A.; Ramos, C.; Neng, N.R.; Nogueira, J.M.F.; Saraiva, J.A.; Nunes, M.L. Chemical composition and antibacterial and antioxidant properties of commercial essential oils. Industrial Crops and Products 2013, 43, 587-595, doi:https://doi.org/10.1016/j.indcrop.2012.07.069.

7. Dosoky, N.S.; Setzer, W.N. Biological activities and safety of Citrus spp. essential oils. International journal of molecular sciences 2018, 19, 1966, doi:10.3390/ijms19071966.

8. Singh, P.; Shukla, R.; Prakash, B.; Kumar, A.; Singh, S.; Mishra, P.K.; Dubey, N.K. Chemical profile, antifungal, antiaflatoxigenic and antioxidant activity of Citrus maxima Burm. and Citrus sinensis (L.) Osbeck essential oils and their cyclic monoterpene, dl-limonene. Food and Chemical Toxicology 2010, 48, 1734-1740, doi:https://doi.org/10.1016/j.fct.2010.04.001.

9. Jabri karoui, I.; Marzouk, B. Characterization of Bioactive Compounds in Tunisian Bitter Orange (Citrus aurantium L.) Peel and Juice and Determination of Their Antioxidant Activities. BioMed Research International 2013, 2013, 345415, doi:10.1155/2013/345415.

10. Frassinetti, S.; Caltavuturo, L.; Cini, M.; Della Croce, C.M.; Maserti, B.E. Antibacterial and antioxidant activity of essential oils from Citrus spp. Journal of Essential Oil Research 2011, 23, 27-31, doi:10.1080/10412905.2011.9700427.

11. Gursoy, N.; Tepe, B.; Sokmen, M. Evaluation of the chemical composition and antioxidant activity of the peel oil of Citrus nobilis. International Journal of Food Properties 2010, 13, 983-991, doi:10.1080/10942910902927136.

12. Moosavy, M.H.; Hassanzadeh, P.; Mohammadzadeh, E.; Mahmoudi, R.; Khatibi, S.A.; Mardani, K. Antioxidant and antimicrobial activities of essential oil of lemon (Citrus limon) peel in vitro and in a food model. 2017, 4, 42-48.

13. Dixit, A.K.; Dixit, C.M.; Yadav, Y.K.; Tewari, A.T.; Shukla, B.V. Standardization of quality parameters in isolated essential oils of Mentha citrat a of Indian origin: under varied geographical location. Journal of Pharmacognosy and Phytochemistry 2014, 3, 119-121.

14. Değirmenci, H.; Erkurt, H. Relationship between volatile components, antimicrobial and antioxidant properties of the essential oil, hydrosol and extracts of Citrus aurantium L. flowers. Journal of Infection and Public Health 2020, 13, 58-67, doi:10.1016/j.jiph.2019.06.017.

15. Merz, K.M.; Ringe, D.; Reynolds, C.H. Drug Design: Structure- and Ligand-Based Approaches; Cambridge, 2010; ISBN 9780521887236.

16. Silva Cavalcanti, A.B.; Costa Barros, R.P.; de Oliveira Costa, V.C.; da Silva, M.S.; Tavares, J.F.; Scotti, L.; Scotti, M.T. Computer-aided chemotaxonomy and bioprospecting study of diterpenes of the Lamiaceae family. Molecules 2019, 24, doi:10.3390/molecules24213908.

17. Araujo, C.A.; da Camara, C.A.G.; de Moraes, M.M.; de Vasconcelos, G.J.N.; Pereira, M.R.; Zartman, C.E. Chemical composition of essential oils from four Piper species, differentiation using multivariate 
analysis and antioxidant activity. Natural Product Research 2020, 1-4, doi:10.1080/14786419.2020.1774761.

18. Lin, L.-Y.; Chuang, C.-H.; Chen, H.-C.; Yang, K.-M. Lime (Citrus aurantifolia (Christm.) Swingle) essential oils: volatile compounds, antioxidant capacity, and hypolipidemic effect. Foods (Basel, Switzerland) 2019, 8, 398, doi:10.3390/foods8090398.

19. Djenane, D. Chemical profile, antibacterial and antioxidant activity of Algerian Citrus essential oils and their application in Sardina pilchardus. Foods (Basel, Switzerland) 2015, 4, 208-228, doi:10.3390/foods4020208.

20. Santos-Sánchez, N.F.; Salas-Coronado, R.; Villanueva-Cañongo, C.; Hernández-Carlos, B. Antioxidant Compounds and Their Antioxidant Mechanism. In Antioxidants; Shalaby, E., Ed.; IntechOpen, 2019.

21. De Oliveira, T.M.; De Carvalho, R.B.F.; Da Costa, I.H.F.; De Oliveira, G.A.L.; De Souza, A.A.; De Lima, S.G.; De Freitas, R.M. Evaluation of p-cymene, a natural antioxidant. Pharmaceutical Biology 2015, 53, 423-428, doi:10.3109/13880209.2014.923003.

22. Foti, M.C.; Ingold, K.U. Mechanism of inhibition of lipid peroxidation by $\gamma$-terpinene, an unusual and potentially useful hydrocarbon antioxidant. Journal of Agricultural and Food Chemistry 2003, 51, 27582765, doi:10.1021/jf020993f.

23. Fitsiou, E.; Anestopoulos, I.; Chlichlia, K.; Galanis, A.; Kourkoutas, I.; Panayiotidis, M.I.; Pappa, A. Antioxidant and antiproliferative properties of the essential oils of Satureja thymbra and Satureja parnassica and their major constituents. Anticancer Research 2016, 36, 5757-5763, doi:10.21873/anticanres.11159.

24. Guo-Xiang, L.; Zai-Qun, L. Unusual antioxidant behavior of $\alpha$ - and $\lambda$-terpinene in protecting methyl linoleate, DNA, and erythrocyte. Journal of Agricultural and Food Chemistry 2009, 57, 3943-3948, doi:10.1021/jf803358g.

25. Essadik, F.Z.; Haida, S.; Kribii, A.; Kribii, A.R.; Ounine, K.; Habsaoui, A. Antioxidant activity of Citrus aurantium L. var. amara Peel from western of Morocco, identification of volatile compounds of its essential oil by GC-MS and a preliminary study of their antibacterial activity. International Journal of Innovation and Scientific Research 2015, 16, 425-432.

26. Lê, S.; Josse, J.; Husson, F. \{FactoMineR\}: A Package for Multivariate Analysis. Journal of Statistical Software 2008, 25, 1-18, doi:10.18637/jss.v025.i01.

27. R Core Team R: A Language and Environment for Statistical Computing 2019.

28. Kassambara, A.; Mundt, F. factoextra: Extract and Visualize the Results of Multivariate Data Analyses 2017.

29. Wickham, H. ggplot2: Elegant Graphics for Data Analysis; Springer-Verlag New York, 2016; ISBN 978-3319-24277-4.

Sample Availability: Samples of Citrus EOs are available from the authors. 